\title{
Ontogeny of Neutrophil Chemotaxis in Fetal Lambs
}

\author{
RUKMANI RAGHUNATHAN, JUDY FAUST, SABITA MISRA, AND MICHAEL E. MILLER \\ Department of Pediatrics, UCLA and UC Davis Schools of Medicine, Harbor-UCLA Medical Center, \\ Torrance, California 90509 and [M.E.M.] 4301 X Street, Sacramento, California 95817
}

\begin{abstract}
Ontogeny of neutrophil chemotactic response using endotoxin activated adult sheep plasma as a source of complement derived chemotactic factor was examined in fetal lambs of gestational age 120-150 days. (Gestational period in sheep is approximately 150 days.) Neutrophils from fetal lambs of gestational age 120-130 days failed to respond to this chemotactic factor whereas neutrophils from fetal lambs above 131 days of gestational age responded at levels comparable to adult values. Examination of neutrophil chemotaxis in older fetuses using a different chemotactic factor derived from mitogen stimulated adult mononuclear cells revealed a selective failure of fetal neutrophils to respond to lymphocyte-derived chemotactic factor in the presence of a normal response to complement derived chemotactic factor. Among prematurely delivered twin fetuses alterations in comparison with the first twin or age-matched controls in peripheral neutrophil count (increase) and in chemotaxis (increase or decrease) were noted in second of twins delivered $\geq 20 \mathrm{~min}$ after the first lamb, suggesting an extreme sensitivity of neutrophil functions to a variety of influences, similar to that seen in humans. (Pediatr Res 20: 265-268, 1986)
\end{abstract}

\section{Abbreviations}

EAP, endotoxin-actived adult sheep plasma

N-LDCF, neutrophil-directed, lymphocyte-derived chemotactic factor

RPMI-FCS, RPMI-1640 with $10 \%$ fetal calf serum

PMN, polymorphonuclear cells

Directed migration or chemotaxis of phagocytes induced by various chemotactic agents is an essential component of inflammatory response. Defects in chemotaxis are associated, as a clinical consequence, with an increased susceptibility to infections. Defects in phagocyte chemotaxis may be due to membrane defects, reduced number of receptors for the chemotactic agent, or to cytoskeletal abnormalities. The chemotactic defect of human neonatal phagocyte originally described by Miller (1) may be due to deficiencies in any or all these components (2-5). Ontogeny of some phagocytic functions, but not chemotaxis, has been evaluated in fetal and neonatal animal models $(6,7)$. The ovine fetus has been shown by us and other investigators to be an appropriate animal model to study the ontogeny of various

Received June 21, 1985; accepted November 6, 1985

Reprints Rukmani Raghunathan, M.D., Department of Pediatrics, HarborUCLA Medical Center, 1000 West Carson Street, E-6, Torrance, CA 90509.

Aided by a grant from the California Research and Education fund of the American Lung Association of California and by BRSG-RR 05551 awarded by the Biomedical Research Support Grant Program, Division of Research Resources, National Institutes of Health. biological phenomena $(8,9)$. In this report, we describe the developmental sequence of neutrophil chemotaxis in fetal lambs, utilizing EAP as a source of complement-derived chemotactic factor. Results from simultaneous evaluation of neutrophil chemotactic responses to EAP and another chemotactic factor derived from mitogen-stimulated adult ovine mononuclear leukocyte (N-LDCF) in a limited number of fetuses are presented In addition, neutrophil counts and chemotaxis in normal fetal lambs and their twins have been compared to document alterations that may be due to "stress" of delayed delivery.

\section{MATERIALS AND METHODS}

Sample collection. Normal adult sheep were used as controls. Heparinized $(10 \mathrm{U} / \mathrm{ml})$ blood was collected from the external jugular vein. Lambs of gestational age 120-150 days and postnatal age 1-6 days were compared with adults. The gestational age was determined by time-dated breeding. The fetal lambs were normal and were being delivered prematurely for other studies. The fetuses were exposed by hysterotomy under maternal spinal anesthesia and heparinized blood was obtained from cord vessels. Results from single fetuses were used to derive developmental data. For twin studies, the second of twins was delivered at varying intervals $(10-40 \mathrm{~min})$ after delivery of the first fetus. During this period, uterine horn containing the fetus and abdominal cavity were covered with warm sterile towels. Term animals were spontaneously delivered and heparinized blood was obtained from a leg vein. The blood was processed within $30 \mathrm{~min}$.

Cell separation. Cell separation was carried out by counterflow centrifugation elutriation technique as previously described (10). In brief, buffy coat leukocytes were resuspended in $0.85 \%$ saline with $0.05 \%$ gelatin and $0.1 \%$ bovine serum albumin and loaded into the separation chamber of an elutriator rotor (Beckman Instruments, Inc., Palo Alto, CA). Red cells and mononuclear cells were removed by stepwise increment in the flow rate at a constant rotor speed for adult blood and by decreasing rotor speed for fetal blood. The granulocytes were retained in the separation chamber. The isolated PMN were $>98 \%$ viable by trypan blue exclusion. Cell yield from peripheral blood averaged $90 \%$, and we were able to obtain $1.0-1.3 \times 10^{6} \mathrm{PMNs}$ from 10 $\mathrm{ml}$ of blood from a 120-day-old lamb.

Preparation of chemotactic factors. Two different chemotactic factors were used in this study. In preliminary experiments, ovine neutrophils were shown not to respond by chemotaxis to the chemotactic peptide, F-Met-Leu-Phe, or human complementderived chemotactic factors. Chemotactic factor derived presumably from sheep complement was prepared by incubating $50 \mu \mathrm{g} /$ $\mathrm{ml}$ of endotoxin (lipopolysaccharide, Sigma Chemicals) with pooled fresh-frozen normal adult sheep plasma for $30 \mathrm{~min}$ at $37^{\circ} \mathrm{C}$ followed by heat inactivation at $56^{\circ} \mathrm{C}$ for $30 \mathrm{~min}$. This preparation is referred to as EAP. In preliminary experiments, $20 \%$ EAP in RPMI-FCS was determined to elicit the maximum chemotactic response in adult sheep neutrophils. Subsequent experiments were done using 10, 20, and 25\% EAP. 
$\mathrm{N}$-LDCF was prepared by incubation of normal adult sheep mononuclear leukocytes $\left(2 \times 10^{6}\right.$ lymphocytes $/ \mathrm{ml}$ in RPMIFCS) with $1 \mu \mathrm{g} / \mathrm{ml}$ of phytohemagglutinin for $48 \mathrm{~h}$ at $37^{\circ} \mathrm{C}$. Supernatants were filtered and frozen at $-70^{\circ} \mathrm{C}$ in aliquots. Control cultures without mitogen were treated similarly and phytohemmagglutinin added at the end of the culture. Optimum concentration of lymphocyte supernatants to demonstrate $\mathrm{N}$ LDCF activity was determined in preliminary experiments with adult ovine neutrophils. The paucity of neutrophils in peripheral blood of fetal lambs precluded testing over a wide range of concentrations of EAP or N-LDCF but at least three concentrations were tested.

Chemotaxis. PMN were resuspended at $1 \times 10^{6} \mathrm{PMN} / \mathrm{ml}$ in RPMI-FCS. Ovine neutrophil chemotaxis was performed in acrylic blind-well chambers (Biorad, Richmond, CA) as previously described by us (9). Briefly, $0.2 \times 10^{5}$ neutrophils in 0.2 $\mathrm{ml}$ were separated from the chemotactic factor or buffer by a 3 $\mu \mathrm{m}$ pore-size polycarbonate chemotactic filter. After incubation for $45 \mathrm{~min}$ at $37^{\circ} \mathrm{C}$ in humidified atmosphere, the filters were stained with hematoxylin and Wright's stain. The number of PMN that had migrated across the filter were counted under an oil immersion lens (magnification $\times 1000$ ) in 10 fields in triplicate filters and results were reported as PMN/field. Net chemotaxis was defined as PMN/field with EAP or stimulated supernatant minus PMN/field with buffer or unstimulated supernatant. Previous experience with these $15 \mu \mathrm{m}$ thickness chemotaxis filters has shown that incubation periods longer than $45 \mathrm{~min}$ resulted in neutrophils being lost from the filter into the lower chamber.

Statistical method. Results were analyzed with $t$ tests using Bonferroni's multiple comparison procedure (11).

\section{RESULTS}

Neutrophil chemotaxis with EAP as chemoattractant. Fetuses were divided into three groups according to their gestational ages (120-130, 131-140, and 141-150 days). Random migration, as defined by PMN/field with buffer or unstimulated lymphocyte supernatant, of adult neutrophils was $3.1 \pm 1.2$ (mean $\pm \mathrm{SD}$ ). Random migration of neutrophils from the three groups of fetal or $2-6$ days postnatal lambs $(2.0 \pm 1.3 ; 2.1 \pm 1.2 ; 2.0 \pm 1.4$ and $2.9 \pm 1.4$, respectively) were comparable to adult values. Directed migration as represented by net chemotaxis ( $=\mathrm{PMN} /$ field with EAP minus PMN/field with buffer) of neutrophils from the four groups of lambs and adults are shown in Figure 1. Neutrophil

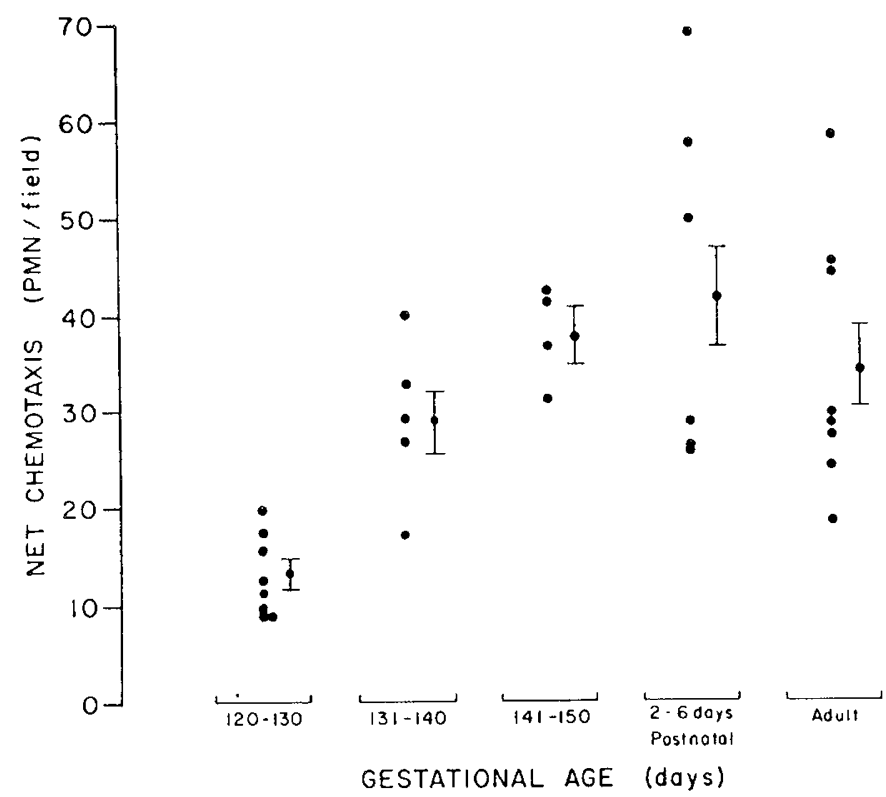

Fig. 1. Ovine neutrophil chemotaxis. Net chemotaxis $=\mathrm{PMN} /$ field with EAP - PMN/field with buffer. Bars indicate SE. chemotaxis in fetuses of $120-130$ days gestational age (mean \pm S.E. $13.4 \pm 1.5)$ was significantly lower than that in the adults $(34.8 \pm 4.4)(p<0.005)$ at $20 \% \mathrm{EAP}$, which concentration elicited the maximum response in general. A similar decrease in chemotaxis was seen with the other concentrations tested. Neutrophil chemotaxis in fetuses of gestational age 131-140 and 141-150 days and in term postnatal lambs (29.3 $\pm 3.6,38.6 \pm$ 4.9 , and $42.2 \pm 15.4$, respectively) were comparable to adult values.

Chemotactic response to $N-L D C F$. Neutrophils from four fetuses were tested for chemotaxis with a different chemoattractant, $\mathrm{N}$-LDCF. Results in Table 1 represent net chemotactic values with $20 \%$ lymphocyte supernatant or $20 \%$ EAP, concentrations which elicited maximal response. Neutrophils from 138- and 148-day fetuses, while responding well to EAP, responded minimally or failed to respond to N-LDCF.

Comparison of neutrophil counts and chemotaxis in twin fetuses. Four sets of twins were available for such comparison. Neutrophil yields from a given volume of blood (as a measure of neutrophil counts) and chemotactic values in these sets of twins are shown in Table 2. Neutrophil yield and chemotaxis were within the normal range for age in the firstborn of all the four sets. Among the second born, one was delivered $10 \mathrm{~min}$ after the first twin. Neutrophil yield and chemotaxis in this second of twins were comparable to those in the corresponding first twin. Second born in three other sets of twins were delivered $\geq 20$ min after the first and neutrophil yields in these lambs were significantly higher than in the corresponding firstborn or agematched controls. In two of these lambs, chemotaxis was also significantly higher $(\mathrm{PMN} /$ field $=20.0$ and 27.7$)$ compared to the first twin (9.0 and 12.5) or age-matched controls (13.4). The third lamb in this group of second twins was delivered $40 \mathrm{~min}$ after the first twin and had the highest neutrophil yield $(5.4 \times$ $10^{6} / 10 \mathrm{ml}$ ), but these neutrophils failed to respond by chemotaxis

Table 1. Neutrophil chemotaxis with EAP and N-LDCF in fetal lambs

\begin{tabular}{cccccc}
\hline & \multicolumn{3}{c}{ Chemotactic response } \\
\cline { 2 - 3 } $\begin{array}{c}\text { Gestational } \\
\text { age of fetus (days) }\end{array}$ & \multicolumn{2}{c}{ Fetus } & & \multicolumn{2}{c}{ Adult } \\
\cline { 2 - 3 } \cline { 5 - 6 } & N-LDCF & EAP & & N-LDCF & EAP \\
\hline 120 & 0.3 & ND* & & 10.2 & 25.8 \\
138 & 1.2 & 20.1 & & 12.4 & 30.2 \\
138 & 4.3 & 22.4 & & 10.5 & 29.4 \\
148 & 1.9 & 56.5 & & 11.0 & 38.7 \\
\hline
\end{tabular}

* Not done.

Table 2. Neutrophil Counts and Chemotaxis in Fetal Lamb Twins

\begin{tabular}{cccc}
\hline $\begin{array}{c}\text { Gestational } \\
\text { age (days) }\end{array}$ & $\begin{array}{c}\text { Time between } \\
\text { delivery of twins } \\
\text { (min) }\end{array}$ & $\begin{array}{c}\text { PMN yield } \\
\text { from } 10 \mathrm{ml} \text { blood } \\
\times 10^{6}\end{array}$ & $\begin{array}{c}\text { Net chemotaxis } \\
\text { (PMN/field) }\end{array}$ \\
\hline $\begin{array}{l}130 \\
\text { Twin 1 }\end{array}$ & & $1.2(1.1)^{*}$ & $15.8(13.4)$ \\
$\quad$ Twin 2 & 10 & 1.8 & 17.8 \\
120 & & $1.0(1.1)$ & $9.0(13.4)$ \\
Twin 1 & 20 & 3.2 & 20.0 \\
Twin 2 & & & $12.5(13.4)$ \\
120 & & $0.9(1.1)$ & 27.7 \\
Twin 1 & 20 & 3.6 & \\
Twin 2 & & & $25.8(29.3)$ \\
132 & & $1.4(1.1)$ & 1.3 \\
Twin 1 & 40 & 5.4 & \\
Twin 2 & 40 &
\end{tabular}

* Nos. in parentheses represent mean values from age-matched controls. 
to a wide range of concentrations of EAP or N-LDCF. Arterial blood gas and $\mathrm{pH}$ determinations failed to reveal any significant alterations in these lambs.

\section{DISCUSSION}

The gestational period in the sheep is approximately 150 days and the period in which the fetal lambs were tested corresponds to the gestational period between $32-40 \mathrm{wk}$ in humans, according to the age equivalence curve developed by Solomon (12).

These results suggest that during normal development, fetal ovine neutrophils respond poorly to complement-derived chemotactic factors until approximately 130 days of gestational age. Functional maturity as defined by chemotaxis comparable to adult levels is attained after this age. This period also corresponds to the appearance of neutrophils in the peripheral blood of the fetus (13).

Neutrophils in the peripheral blood of unstressed 120- to 130day fetuses were morphologically similar to those of older fetuses or adults. Although elutriation profiles (flow rates and centrifugal force required for elution) of fetal leukocytes and red cells in general were different from that of adult cells, leukocytes of all groups of fetuses (120-150 days) had a similar profile. Thus we were unable to identify an obviously immature neutrophil as being responsible for the chemotactic defect. The chemotactic defect of fetal ovine neutrophils is unlikely to be related to chemotactic factor inactivator documented in the 110-120 days of gestational age lambs since chemotactic factor inactivator has been shown not to be directed against neutrophils (14).

A similar developmental delay in chemotaxis of human neutrophils has been described, although in humans this "defect" may persist for the first few years of life $(15,16)$. In the human neonate, factors suggested to be responsible for the defect include deficiency of membrane receptors, cytoskeletal abnormalities, and membrane rigidity $(2-5,17)$. We have previously shown that chemotaxis of neutrophils obtained from human cord blood is comparable to adult values and "increased" in comparison to chemotaxis of neutrophils from same infant $48-72 \mathrm{~h}$ after birth (18). Similarly, the present study has documented "normal" values in 120 day gestational age "stressed" (delivered 20 min after the first) lambs whereas their unstressed twins had significantly decreased chemotaxis. These data suggest that ontogenic delay in the appearance of complement receptors is unlikely to be the only reason for the neutrophil chemotactic defect of the fetus or newborn in both humans and sheep. Sequential analysis of fetal ovine neutrophil cytoskeletal structure and functions and membrane fluidity in comparison with human neutrophils will define the role of these components in the ontogeny of cell movement.

Observations of increased peripheral neutrophil counts in cord blood and peripheral blood soon after birth in humans and sheep (18, 20, Raghunathan R, Miller ME, unpublished observations) are similar to the limited observations in the present study of increased neutrophil counts in the "stressed" preterm lambs compared to the corresponding firstborn. In humans, these numerical alterations are associated with functional alterations (increase in chemotaxis and decreased oxidative metabolic activity) (18-21; Raghunathan R, Miller ME, unpublished observations). The transient nature of these alterations has been documented by the fall in neutrophil counts and chemotaxis in the subsequent $48-72 \mathrm{~h}$ in humans and by the fall in peripheral PMN counts in neonatal lambs. Observations of increased peripheral neutrophil counts and decreased neutrophil oxidative metabolic activity in cord blood of infants delivered vaginally or by cesarian section following labor compared to that of infants delivered by cesarian section without labor $(20,21)$ related such numerical and functional alterations to the process of labor which may be associated with release of various mediators. Alterations in neutrophil counts and chemotaxis seen in the "stressed" preterm fetuses in comparison to their unstressed twins may be due to a similar phenomenon of a lesser degree, although this "stress" may not be strictly comparable to the stress of labor and vaginal delivery. The failure of neutrophils from 132-day gestational age second twin delivered $40 \mathrm{~min}$ after the first to respond by chemotaxis is reminiscent of defective chemotaxis seen in severely infected and stressed infants and may reflect a later stage in the continuum of stress-related alterations in neutrophil function due either to receptor modulation or intrinsic defect in the cellular movement mechanism.

Observations from the twin study exemplifies the sensitivity of neutrophil functions to various modulating influences and underscores the need for caution in the interpretation of results of neutrophil functional studies in clinical settings. These observations, although not conclusive because of small sample size, suggest that numerical and functional alterations of neutrophils due to stress-related mediators may be similar in sheep and humans. Further studies comparing neutrophil functions in surgically and vaginally delivered lambs are necessary for confirmation of these observations and for elucidation of the role of stress and maternal/fetal mediators on neutrophil functions. Such studies will also establish the usefulness of fetal lamb as a model to examine the neonatal inflammatory response.

Failure of fetal ovine neutrophils to respond to N-LDCF was unexpected in view of their normal response to EAP. This failure may be due to an ontogeneic delay in the appearance of LDCF receptors of ovine neutrophils. Our observation that neutrophils from two normal pregnant ewes also failed to move in response to N-LDCF while maintaining a normal response to EAP has suggested an alternate explanation. Fetal neutrophils may have undergone modulation of LDCF receptors due to in vivo exposure to N-LDCF liberated during maternal-fetal interactions. Lymphocyte derived chemotactic factors have been demonstrated during in vitro allogeneic reactions (22) and in vivo in inbred rats carrying heterozygous pregnancies (23). We have previously demonstrated a failure of N-LDCF production by mitogen-stimulated fetal mononuclear leukocyte (9). Fetal mononuclear leukocyte production of N-LDCF during alloreactions has not yet been thoroughly tested in this species. It is possible that N-LDCF of maternal or fetal origin may deactivate fetal neutrophils. Human cord monocytes obtained from term, vaginally delivered neonates have been shown to respond to LDCF (24), but these phagocytes may differ in their functional capability from cells of neonates who have recovered from the effects of labor and delivery. Lymphokine-mediated phagocyte accumulation may be an essential effector component of in vivo cell-mediated immune response. Deficient lymphokine-mediated inflammatory response during intrauterine period may contribute toward maintenance of an allogeneic pregnancy and thus would be of teleologic significance. Studies evaluating human monocyte and PMN chemotactic response to LDCF in the neonatal period are not available and such studies would contribute significantly toward understanding of the ontogeny of inflammatory response.

In summary, we have described the ontogenic sequence of neutrophil chemotaxis to complement-derived chemotactic factors and to lymphokine in fetal lambs. Similarities in neutrophil homeostatic mechanisms of sheep and humans observed in this study suggests that the fetal lambs may be an excellent animal model for studying the ontogeny of inflammatory response.

Acknowledgments. The authors acknowledge with gratitude the help of Drs. Jobe, Ikegami, Padbury and Klein of the Division of Neonatology, Harbor-UCLA Medical Center, in obtaining cord blood samples of fetal lambs and the patient secretarial help from Joy Heiner.

\section{REFERENCES}

1. Miller ME 1971 Chemotactic function in the human neonate: humoral and cellular aspects. Pediatr Res 5:487

2. Mease AD, Fischer GW, Hunter KW, Ruymann FB 1980 Decreased phytohemagglutinin-induced aggregation and C5a-induced chemotaxis of human newborn neutrophils. Pediatr Res 14:142-146 
3. Anderson DC, Hughes BJ, Smith CW 1981 Abnormal mobility of neonatal polymorphonuclear leukocytes. Relationship to impaired redistribution of surface adhesion sites by chemotactic factor or colchicine. J Clin Invest 68:863-874

4. Nunoi H, Endo F, Chikazawa S, Namikawa T, Matsuda I 1983 Chemotactic receptor of cord blood granulocytes to the synthesized chemotactic peptide N-formyl-methionyl-leucyl-phenylalanine. Pediatr Res 17:57-60

5. Kimura GM, Miller ME, Raghunathan R. Leake RD 1981 Reduced concanavalin-A capping of neonatal PMNs. Pediatr Res 15:1271-1273

6. Zeligs BJ. Nerurkar LS, Bellanti JA 1977 Maturation of the rabbit alveolar macrophage during animal development III. Phagocytic and bactericidal functions. Pediatr Res 11:1208-1211

7. Reade PC, Jenkin CR 1965 The functional development of the reticuloendothelial system. Immunology 9:53-60

8. Granberg E, Hirvonen $T 1980$ Cell-mediated lympholysis by fetal and neonatal lymphocytes in sheep and man. Cell Immunol 51:13-22

9. Raghunathan R, Miller ME, Wuest C, Faust J 1984 Ontogeny of the immune system. Fetal lamb as a model. Pediatr Res 18:451-456

10. Raghunathan R, Wuest C, Faust J, Hwang S, Miller ME 1982 Isolation of ovine lymphocytes, granulocytes and monocytes using counterflow centrifugation-elutriation. Am J Vet Res 43:1467-1470

11. Miller RG 1981 Normal univariate techniques. In: Simultaneous Statistical Inference. Springer-Verlag, New York, pp 67-70

12. Solomon JB 1971 The unification of foetal and post-natal immunology in mammals and birds by age equivalence. In: Foetal and Neonatal Immunology, Elsevier Publishing Co., Inc., New York, pp 343-361

13. Sawyer M, Moe J, Osburn BI 1978 Ontogeny of immunity and leukocytes in the ovine fetus and elevation of immunoglobulins related to congenital infection. Am J Vet Res 39:643-648

14. Tannous R, Kisker CT, Clarke WR 1982 Development of chemotactic factor inactivation-a fetal lamb model. Pediatr Res 16:538-542

15. Klein RB, Fischer TJ, Gard SE, Biberstein M, Rich KC, Stiehm ER 1977 Decreased mononuclear and polymorphonuclear chemotaxis in human newborns, infants and young children. Pediatrics 60:467-472

16. Yegin O 1983 Chemotaxis in childhood. Pediatr Res 17:183-187

17. Miller ME, Myers KA 1977 Membrane deformability of human polymorphonuclear leukocytes and its relationship to developmental, normal and abnormal PMN movement. In: Cooper MD, Dayton DH (eds) Development of Host Defenses. Raven Press, NY, pp 175-185

18. Raghunathan R, Miller ME, Everett S, Leake RD 1982 Phagocyte chemotaxis in the perinatal period. $J$ Clin Immunol 2:242-245

19. Schulman I 1972 Diseases of the white blood cell. In: Barnett HL (ed) Pediatrics. Appleton-Century Crofts, New York, p 1203

20. Frazier JP, Cleary TG, Pickering LK, Kohl S, Ross PJ 1982 Leukocyte function in healthy neonates following vaginal and cesarian section deliveries. $\mathrm{J}$ Pediatr 101:269-272

21. Dalens BJ, Travade PH 1983 Effect of mode of delivery on leukocyte function of neonates. J Pediatr 102:1014

22. Snyderman R, Meadows L and Pike MC 1979 Quantification of lymphokine production in human disease. In: Cohen S, Pick E, Oppenheim J (eds) Biology of the Lymphokines. Academic Press, New York, pp 181-208

23. Gill TJ III, Rabin BS, Junz HW, Davis BK, Taylor FH 1977 Immunological aspects of maternal-fetal interactions. In: Cooper MD, Dayton DH (eds) Development of Host Defenses. Raven Press, New York, pp 297-302

24. Berman JD, Johnson WD 1978 Monocyte function in human neonates. Infect Immun 19:898-902 